\title{
Researching of quasiperiodic variations in the amplitude of VLF electromagnetic signals from lightning discharges passing over strong earthquakes
}

\author{
Vyacheslav Argunov ${ }^{a l}$ and Mikhail Gotovtsev ${ }^{a}$ \\ ${ }^{a}$ Yu.G. Shafer Institute of Cosmophysical Research and Aeronomy of the Siberian Branch of the \\ Russian Academy of sciences, Yakutsk, Russian Federation
}

\begin{abstract}
The specific features of a method for radiosounding the lower ionosphere over earthquake epicenters using LF electromagnetic signals of thunderstorm sources (atmospherics) have been considered. The effects of shallow-focus earthquakes with magnitudes larger than 4.0 and their precursors manifest themselves in amplitude characteristics of atmospherics. It has been assumed that variations in the signal characteristics are related to disturbances in the lower ionosphere. According to the results of azimuthal scanning, cross sectional dimensions of disturbed regions, as a rule, correspond to the dimensions of the first two Fresnel zones for signals at a frequency of $10 \mathrm{kHz}$. Azimuthal scanning also indicated that the positions of disturbed regions during and before earthquakes could have a certain dynamics and differ from the projection onto the earthquake epicenter. Quasi-periodic variations of signal amplitude of lightning discharges passing over earthquake epicenters are in details considered. It has been obtained that the period of amplitude modulation makes up 2-3 hours, i.e. in the ionosphere D-layer the same values of the periods of wave phenomena are registered as which are observed in a higher F-region of the ionosphere. The results obtained during the work specify that, at least, in the part of events the seismic disturbances in the ionosphere can be caused by AGW, propagating upwards from the epicentral area.
\end{abstract}

\section{Introduction}

The investigation of manifestations of lithospheric processes in the lower ionosphere is usually done using the signals of very-low-frequency (VLF) or low-frequency (LF) radio transmitters [1-6]. The presence of disturbances in the ionosphere caused by seismic events is confirmed in a number of papers (see, e.g. [7-9]). As it is known, the most sensitive parameter of VLF signal is its phase. Amplitude measurements refer to the rougher methods, although it should be noted that in a number of papers [4,5], the amplitude measurements are used in the search of ionospheric precursors of strong earthquakes (EQs), but those measurements are based on the interference (though on the phase relations) of electromagnetic waves in the vicinity of the terminator. As an alternative, or supplementing method for detection of seismic disturbances in the ionosphere, the use of natural radioelectromagnetic emission of lightning discharges - atmospherics can be considered. This method gives the possibility to conduct a monitoring scan of a large seismically active region or of several regions directly from one point. At the same time, this modification of

1 Corresponding author: vvargunov@ikfia.ysn.ru 
the method has its own particular qualities. In this paper, these features are considered in relation to the receiving point in the city of Yakutsk.

\section{Method used to measure and analyse the parameters of atmospherics}

Receiving the signals of atmospherics is carried out at Yakutsk $(\varphi=62.02 \mathrm{~N}, \lambda=129.71 \mathrm{E})$ with the one-point lightning pelengator-range finder whose characteristics are corrected with reference to the registration of distant atmospherics. The signals of atmospherics are received with three antennas: an electrical (dowel) and two orthogonal magnetic antennas (loops with effective square of $180 \mathrm{~m} 2$ ). During winter time at the receiving point the maximum distance up to the sources of received signals (determined by a possibility of extraction from noisy signals) is about $12000 \mathrm{~km}$. However, the distance up to the epicenter of lithospheric disturbances revealed confidently through the lower ionosphere on the path of atmospheric propagation as our studies showed earlier 13, is restricted by values of $3500-4000 \mathrm{~km}$. At distances over $4000 \mathrm{~km}$ the amount of variations in the atmospherics amplitude increases. The earthquake manifestations in the signals of atmospherics are usually analyzed for night conditions when a signal attenuation in the "earth-ionosphere" waveguide is the smallest. At the same time taking into account the interference of signal modes at nights complicating the character of amplitude variations, also the day-to-day variations of the amplitude determined during the day hours are additionally considered. However, during the day hours the number of registered atmospherics due to signal attenuation in waveguide is considerably reduced in comparison with night conditions and, correspondingly, errors of determination of averaged amplitude values increase.

For today, several mechanisms have been proposed for transferring the energy of seismic processes to ionospheric heights. One of the mechanisms is based on the possible infrasonic coupling of lithospheric and ionospheric disturbances in times of earthquakes and during periods of their preparation [10]. Another possible mechanism [11] is widely considered, it based on the interrelationship between lithospheric-atmospheric and electromagnetic processes (air ionization processes, transferring of electric fields to ionospheric heights). According to above characteristics of ionospheric disturbances associated with seismic phenomena, much attention is paid to the mechanism in which as a source of disturbances in the ionosphere is considered acoustic-gravity waves (AGW) caused by lithospheric processes. In the AGW spectrum, internal gravity waves (IGW) [12] are distinguished, the feature of which is the presence of a transverse component of the propagation velocity in them. A number of works indicate the possibility of excitation of ionospheric disturbances by AGW (IGW), generated by strong seismic events [12]. Modeling the propagation of AGW from impulse ground sources (earthquakes) confirms this possibility [13].

In the case of wave mechanisms, one should expect the presence of modulation effects in the parameters of ionospheric disturbances. In particular, in [14], is noted for 1-2 days before the earthquakes a more frequent occurrence of long-period (with a characteristic time on the order of $2 \mathrm{~h}$ ) quasi-wave disturbances in the spectrum of density variations of F-layer.

In the present work, such possible effects are considered at D-layer heights on the basis of remote sensing data of seismic disturbances in the lower ionosphere by using the natural electromagnetic signals of thunderstorm origin (atmospherics) according to the method described in [15]. 


\section{Results}

The quasiperiodic variations in the amplitude of atmospheric's signals during the period of strong seismic activity in the area of the Japanese island of Honshu in March 2011 were reported in [15]. These variations, expressed in the form of train of oscillations with a duration of up to 4-5 periods, were observed both after the strongest earthquake on March 11,2011 with a magnitude of 9 , and before this event. The effects of this earthquake and previous one on 09.03.2011 with magnitude of 7.2 were expressed in the signals of atmospherics in the form of an increase in their amplitude. In February, two more cases of amplification of atmospherics were recorded, which are considered in [15] as precursors of these earthquakes. The search for possible precursors of this catastrophic event was also carried out in wave phenomena. So in work [16] internal gravity waves (IGW) were considered upon of variations in atmospheric temperature, pressure and humidity at different heights. It was found that exactly one month before earthquake on 11.03.2011 IGW was observed, with high probability associated with seismic activity (precursor). In this respect, let us begin our consideration of quasiperiodic variations of atmospheric's signals from this strong seismic event.

The general picture of variations in the amplitude of atmospherics in February 2011 is shown in Fig. 1a.
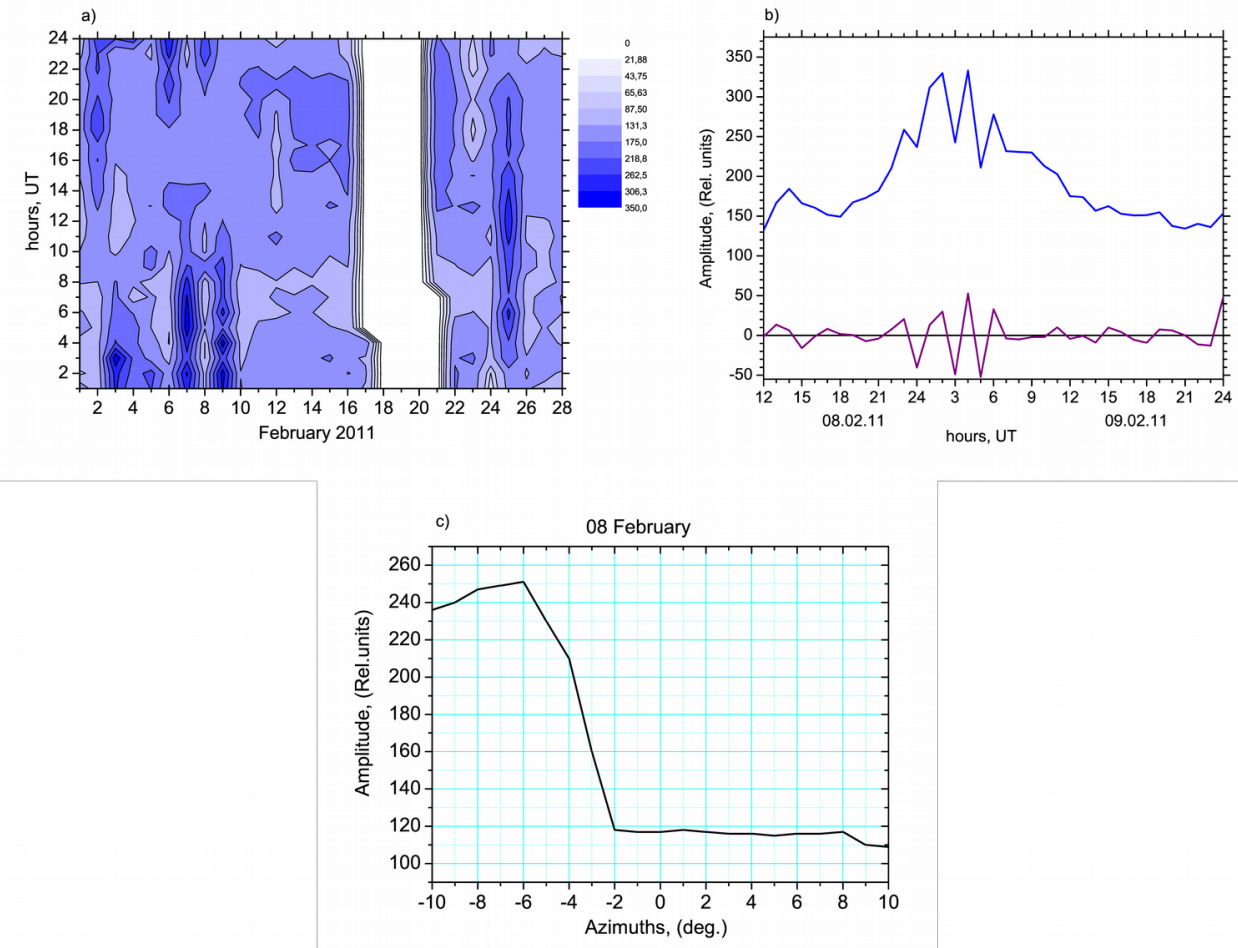

Fig 1. (a) Variations of the amplitude of lightning discharges in February 2011 at all hours of the day. Variations of amplitude are shown by color intensity, white color - no data (February 17-22); (b) atmospheric amplitude variations on February 8 and 9; (c) azimuthal distribution of atmospheric amplitudes from directions on earthquake. 
Here, the amplitude is represented in gradations of color (the scale is shown on the right). Significant increase in amplitude with duration of some hours observed 02, 06-07, 08-09 and 25 February. The latter increase noted in [15] as one of the precursors of earthquakes. It can be seen that the increase of the amplitude has a modulated character. More details of the variations on 08-09 February are shown in Fig. 1b (a train of quasiperiodic variations was observed). The quasiperiod of variations is $2-3$ hours. Note that the analysis uses the hourly averaged values of the amplitude. The fact that this amplitude increasing can be linked to followed a month later earthquakes, indicates the presence of azimuthal region of the maximum of amplitude increase approximately coinciding with the position of the epicenter (Fig. 1c shows the azimuthal distribution of the signal amplitude in the 01 UT February 8, 2011 with respect to the azimuth of the epicenter of the earthquake - value " 0 " along the ordinate axis). The maximum of the azimuthal distribution of the amplitude is somewhat shifted toward higher latitudes, which may well be, taking into account that the observed features of the amplitude variations of the signals reflect the changes in the medium (plasma) at ionospheric altitudes.

At the same time, the expected variation in the period on February 10-12 (in accordance with the results of [16]) cannot be detected.

Let us consider another monthly interval with quasiperiodic variations in the amplitude of atmospherics - December 2012. The measurements were made in the angular sector of the Fresnel 5 th zone in the direction of the northern part of the Japanese islands (coordinates of the virtual epicenter: $\varphi=35.5^{\circ} \mathrm{N}, \lambda=137.5^{\circ} \mathrm{E}$ ). Amplitude variations for the whole month are shown in Fig. 2a, where it can be seen that the significant amplitude increases in 4 time periods duration of a few hours were observed $(3,5,7,15$ of December) and one very significant - hourly (at 06 UT of December 10).
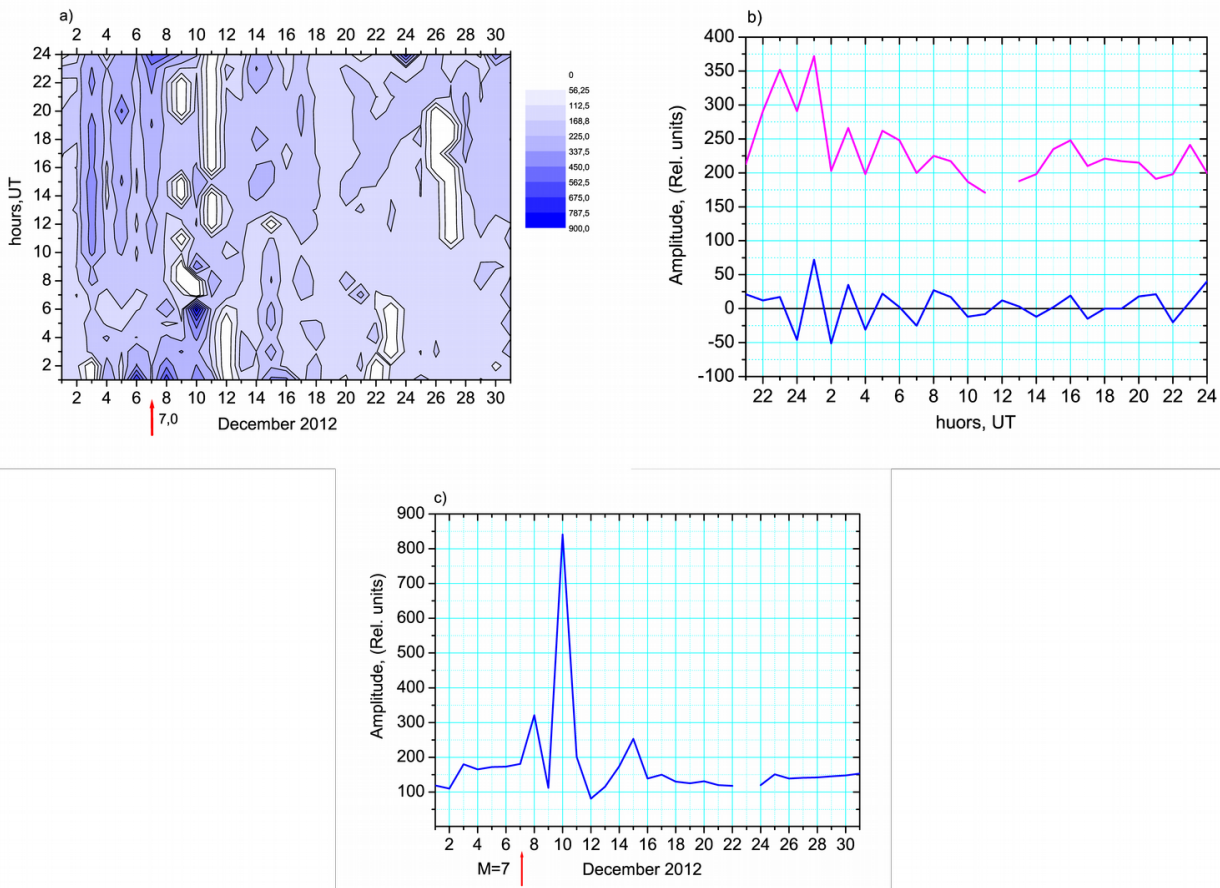

Fig 2. a) Atmospheric amplitude variations in December 2012; b) atmospheric amplitude variations in December 6UT; c) amplitude variations December 15. 
November and December 2012 in the area of the Japanese islands were characterized by high seismic activity, therefore, therefore it is difficult compare these semi-diurnal increases of amplitude with a concrete earthquake (earthquake effect or its precursor). The exception is the event of a very strong hourly increases (more than 5 times, Fig. 2b) on December 10, which according to the results of [2] (the effects of proper earthquakes are observed on the day of the event or on one of the next three days) can be considered as the effect of an earthquake that occurred on December 7 and had a magnitude of 7 . Note that the value of the geomagnetic $\mathrm{Kp}$ index in the cases of these increases in a given month did not exceed 3 (the magnetic field was quiet), which makes it possible to exclude the geomagnetic cause of the ionospheric disturbances with which this strong increases of the amplitude of the atmospherics is related. Expressed quasiperiodic variations of the amplitude («shock» exitation of oscillation) are visible on December 15, which is confirmed by Fig. 2c. The quasi-period of the amplitude oscillation train in this event is also about 2 hours.

\section{Conclusion}

Quasi-periodic variations in the events of increase of the signals of atmospherics caused by seismic disturbances in the lower ionosphere are considered. The predominant amplitude modulation period is 2-3 hours, i.e. in the D-layer of the ionosphere, the same values of the periods of wave phenomena as observed in the higher $\mathrm{F}$ region of the ionosphere are recorded. The obtained values of the modulation periods correspond to the range of periods of internal gravitational waves (IGW). Part of the events clearly expresses the "shock" nature of the phenomenon - a train of damped oscillations of the specified period range. This may indicate that, at least in part of the events, seismic disturbances in the ionosphere may be due to IGW propagating upward from the epicenter region.

This work is supported by Project II.16.2.1 (registration number AAAA-A17117021450059-3)

\section{References}

1. P.F., Biagi, L. Castellana, T. Maggipinto, R. Piccolo, A. Minafra, A. Ermini, S. Martellucci, C. Bellecci, G. Perna, V. Capozzi, O.A. Molchanov, M. Hayakawa, Natural Hazards and Earth System Sciences, 5, 727-732, (2005)

2. P.F. Biagi, R. Piccolo, A. Ermini, S. Martelucci, C. Belecci, M. Hayakawa, V. Capozzi, S.P. Kingsley, Natural Hazards and Earth System Sciences, 1, 99-104, (2001)

3. P.F. Biagi, R. Piccolo, L. Castellana, A. Ermini, S. Martelucci, C. Belecci, V. Capozzi, G. Perna, O.A. Molchanov, M. Hayakawa, Physics and Chemistry of the Earth, 29, 551-557, (2004)

4. M. Hayakawa, O.A. Molchanov, T. Ondoh, E. Kawai, Journal of Atmospheric Electricity, 16, 247-257, (1996)

5. M. Hayakawa, O.A. Molchanov, T. Ondoh, E. Kawai, Journal of Physics of the Earth, 44, 413-418, (1996)

6. A. Rozhnoi, M. Solovieva, O.A. Molchanov, P.F. Biagi, M.Hayakawa, K. Schwingenschuh, M. Boudjada, M. Parrot, Natural Hazards and Earth System Sciences, 10, 529-534, (2010)

7. V.A. Liperovsky, O.A. Pokhotelov, E.V. Liperovskaya, M. Parrot, C.V. Meister, A. Alimov, Surveys in Geophysics, 21, 449-486, (2000)

8. E.V. Liperovskaya, O.A. Pokhotelov, Y. Hobara, M. Parrot, Natural Hazards and Earth System Sciences, 3, 279-284, (2003) 
9. V.A. Liperovsky, C.V. Meister, E.V. Liperovskaya, N.E. Vasil'eva, O. Alimov, Natural Hazards and Earth System Sciences, 5, 59-62, (2005)

10. V.V. Kuznetsov, A.S. Alekseev, Science. - 94, (1992)

11. S.A. Pulinets, D.V. Davidenko, A.D. Linkov, Fundamental problems of electronic instrument making, 12, 7, 57-65, (2012)

12. M.G. Gokhberg, S.L. Shalimov, Electronic Journal "Russian Journal of Earth Sciences", 2, 95-108, (2000)

13. V.E. Kunitsyn, S.N. Suraev, R.R. Akhmedov, Moscow University Bulletin. Series 3. Physics. Astronomy, 2, 59-63, (2007)

14. V.A. Liperovsky, L.E. Kolokolov, E.V. Liperovskaya, Earth Physics, 7, 101-109, (1992)

15. V.A. Mullayarov, V.V. Argunov, L.M. Abzaletdinova, G.I. Druzih, A.N. Mel'nikov, Natural Hazards and Earth System Science, 12, 3181-3190, (2012)

16. V.B. Kashkin, Optics of the Atmosphere and Ocean, 26, 10, 908-916, (2013) 